\title{
Pulse Heat Treatment of FINEMET Alloys Under Tension
}

\author{
A. LOVAS ${ }^{a}$, L. HuBAČ $\check{C}^{b, *}$, L. NOVÁK ${ }^{b}$ \\ ${ }^{a}$ Department of Automobiles and Vehicles, Budapest University of Technology and Economics, \\ Stoczek u .2. 1111, Hungary \\ ${ }^{b}$ Department of Physics, Technical University of Košice, Park Komenského 2, 04200 Košice, Slovakia \\ In this contribution a less common experimental procedure is presented, which includes simultaneous appli- \\ cation of electrical pulse heating, and longitudinal mechanical stress, applied along the ribbon length, for the \\ magnetic property tailoring. The annealing is performed in protecting atmosphere in a vertical tube furnace by \\ applying a weight at the free, cold bottom end of the ribbon. In these circumstances neither nanometer-size grains \\ nor the concentration-distribution have been developed. The static coercive force, anisotropy and demagnetizing \\ factor are monitored as a function of pulse number.
}

DOI: 10.12693 /APhysPolA.126.100

PACS: 75.50.Kj, 75.60.Ej, 81.07.Bc

\section{Introduction}

The nanocrystallization from FINEMET-type amorphous precursors opens a wide-range possibility for the property-tailoring in soft magnetic alloys [1]. Not only the exceptional magnetic softness (minimization of coercive force $\left(\mathrm{H}_{c}\right)$, efficient lowering of power loss $(P)$, but also the shape of magnetization curve can be easily altered in these alloys by applying appropriate field and stress annealings. In the large scale (industrial) production, both the traditional crystallization heat treatments and the stress annealing $[2,3]$ are applied.

\section{Experiment, materials and measurement methods, heat treatments}

Impulse heating was realized by alternating the current pulses of approximately $10 \mathrm{~A}, 50 \mathrm{~Hz}$. Pulse duration was $0.3-0.5 \mathrm{~s}$. Samples were processed in this heat without forced air cooling. Thus, the estimated cooling rate was about $100 \mathrm{~K} / \mathrm{s}$. Thermal heating rate was about $1000 \mathrm{~K} / \mathrm{s}$. The width of the samples was about $10 \mathrm{~mm}$, thickness $-30 \mu \mathrm{m}$ and length $-100 \mathrm{~mm}$. Additional uniaxial stress $(\sigma=0,2000$ and $4000 \mathrm{kPa})$ was applied to FINEMET ribbons. The time-scale (and hence the power of the pulse was regulated electronically. The measurements were performed in a static magnetometer. The coercive field $\left(\mathrm{H}_{c}\right)$, anisotropy $\mathrm{K}_{\sigma}$ and $\mathrm{K}_{i}$, were determined from the magnetization curves, which were plotted after each individual pulse.

\section{Results and discussion}

\subsection{Change of coercive force during the pulse treatments}

In Fig. 1. the drop of $H_{c}$ versus the number of heat pulses is compared. When only magnetic field $H=1000 \mathrm{~A} / \mathrm{m}$ was applied during the pulse treatment (without application of stress), the slope of $H_{c}(n)$ curve

\footnotetext{
*corresponding author; e-mail: lukas.hubac@tuke.sk
}

was small. The reason for this is probably the interaction between the longitudinal and the circumferential (pulse current-induced) field. The $H_{c}$ decreases more rapidly in all other cases, (i.e the slope is higher), and decreases even more when the applied stress is higher (4000 kPa).

One can see, that the evolution of curves is not strictly monotonic, a fluctuation is developed on each of curves, in the range of small pulse numbers $(n \leq 5)$. The amplitude of this fluctuation is higher than the experimental error. This fluctuation seems to disappear as the longitudinal stress increases.

\subsection{The anisotropy change during the pulse treatments}

A similar stepwise character appears in the $\mathrm{K}_{\sigma}$ and $\mathrm{K}_{i}$ when they are plotted versus the pulse number (see Fig. $2 \mathrm{a}$ and $\mathrm{b}$ ).

\section{Discussion}

As Fig. 1. shows, the stress relaxation induced by the heat pulse $\left(\mathrm{H}_{c}\right.$ decrease $)$ has a dominant contribution to the net magnetic softening, compared with the influence of nanocrystalline grain formation itself. This finding is in qualitative agreement with the early results reported in Ref. [4].

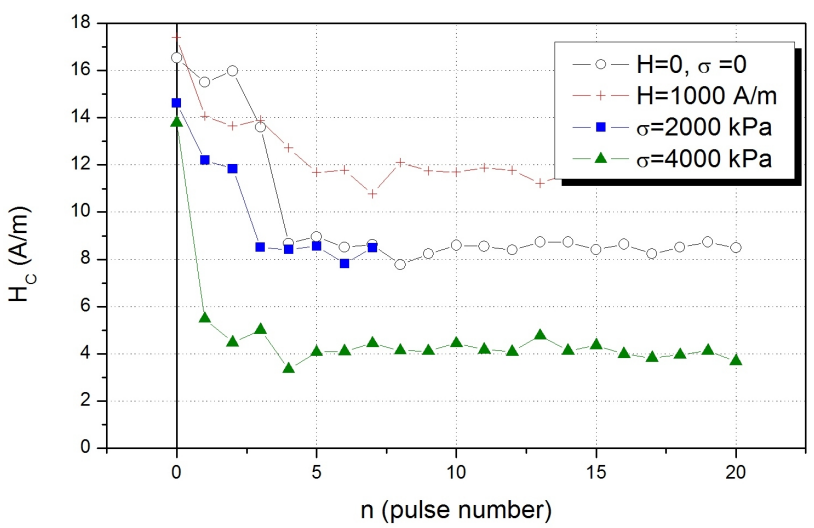

Fig. 1. Variation of coercive force as a function of pulse number. 

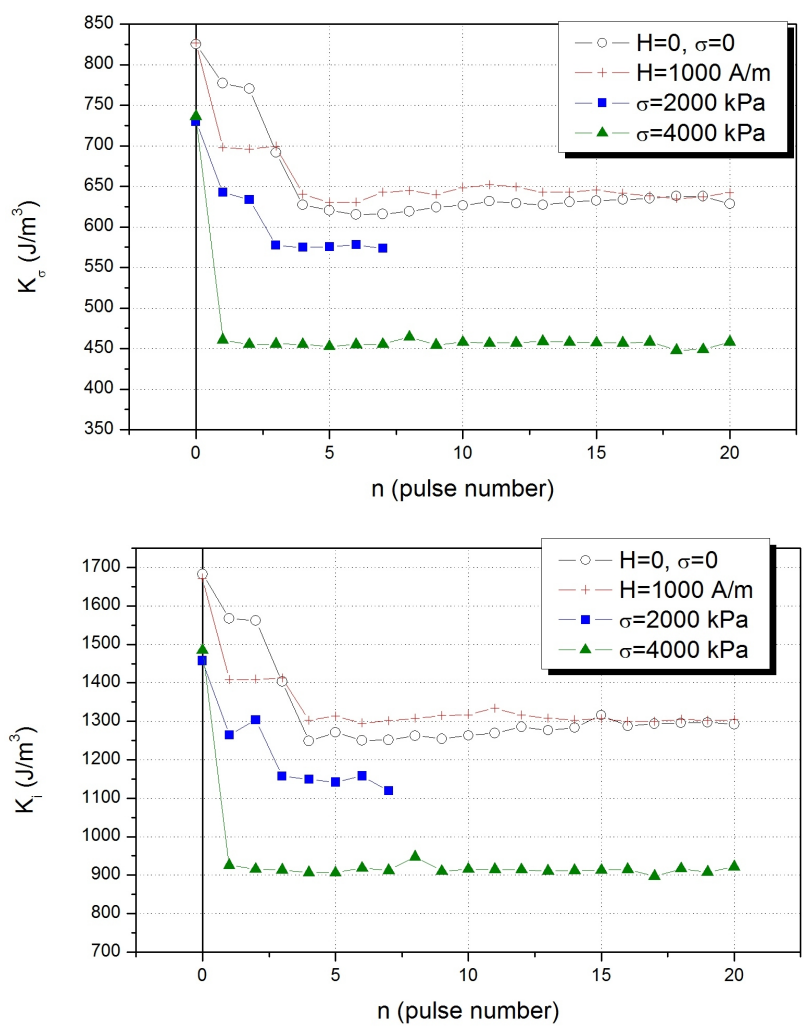

Fig. 2. a) $K_{\sigma}$ versus the pulse number, b) The induced anisotropy $\left(\mathrm{K}_{i}\right)$ versus the pulse number.

\subsection{Demagnetizing factor}

A similar trend can be observed in the behaviour of $D(n)$.The relaxation is highly enhanced by the application of longitudinal stress during the heat treatments. Though a high structural resolution study does not yet support directly this argument, the development of nanocrystalline structure is not plausible during the available short heating period. Rather the stress-induced creep can be the structural background of the observed phenomena. The crystallization requires chemical decomposition of the precursor glass at least in nanometerscale, resulting the well known two-phase structure. As the result of this decomposition, the mechanical brittleness is developed by applying the traditional nanocrystallization technique. Such brittleness is not experienced in the present study.

This finding seems to decrease the explanatory power of random anisotropy model, which postulates pronounced grain-size related background for the dramatic $H_{c}$ decrease in the FINEMET-type soft magnets.

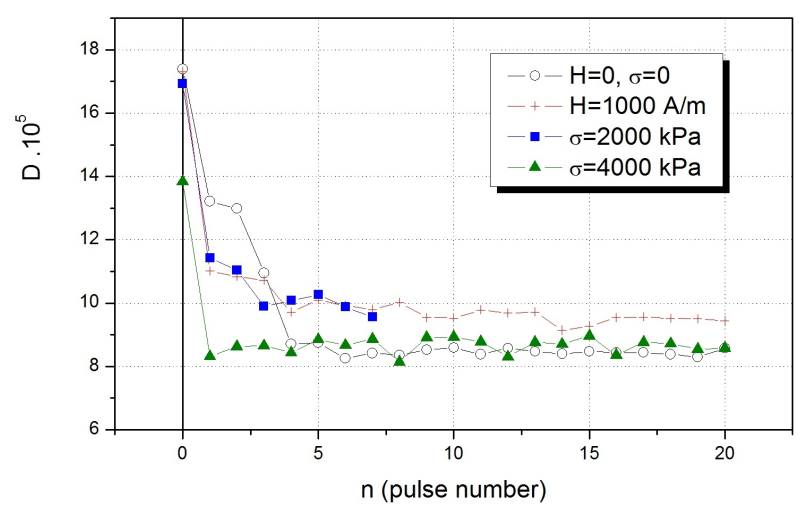

Fig. 3. The change of demagnetizing factor versus heat pulse number.

\section{Conclusions}

1. Significant magnetic softening can be achieved by pulse heat treatments, without nanocrystalline grain formation.

2. The mechanical flexibility of samples is sustained during the whole experiments, no brittle fracture is experienced.

3. Due to the short-time heat pulse, long range diffusion in the samples is arrested, only stress relaxation (elimination of stress centers) may participate in the property changes, however, stress-induced creep cannot be excluded.

4. High resolution structural study is required in order to reveal the structural details of the observed phenomena.

\section{Acknowledgments}

This work was supported by the project OTKA K109271 and also by the project VEGA 1/0778/12.

\section{References}

[1] G. Herzer, Handbook of Magnetic Materials ed. Buschow K.H.J. v.10, chapt. 3. 1997, p.415 Elsevier Science, Amsterdam.

[2] A. Lovas, L.F. Kiss, B. Varga, P. Kamasa, I. Balogh, I. Bakonyi, J. Phys. IV France 8, Pr2-291 (1998).

[3] L.K. Varga, Z. Gercsi, G. Kovács, A. Kákay, F. Mazaleyrat, J. Magn. Magn. Mater. 254-255, 477 (2003).

[4] A. Lovas, B. Varga, Czechoslovak Journal of Physics 52, 155 (2002). 